\title{
BMJ Open Impact of multidrug-resistant tuberculosis and its medications on adverse maternal and perinatal outcomes: protocol for a systematic review and meta-analysis
}

\author{
Kefyalew Addis Alene, ${ }^{1,2,3}$ Akilew Awoke Adane, ${ }^{3}$ Alemken Jegnie (D) ${ }^{4}$
}

To cite: Alene KA, Adane AA, Jegnie A. Impact of multidrugresistant tuberculosis and its medications on adverse maternal and perinatal outcomes: protocol for a systematic review and meta-analysis. BMJ Open 2019;9:e034821. doi:10.1136/ bmjopen-2019-034821

- Prepublication history and additional material for this paper are available online. To view these files, please visit the journal online (http://dx.doi. org/10.1136/bmjopen-2019034821).

Received 08 0ctober 2019 Accepted 25 November 2019

Check for updates

(C) Author(s) (or their employer(s)) 2019. Re-use permitted under CC BY-NC. No commercial re-use. See rights and permissions. Published by BMJ.

${ }^{1}$ Health Sciences, Curtin University Bentley Campus, Perth, Western Australia,

Australia

${ }^{2}$ College of Medicine and Health Sciences, University of Gondar, Gondar, Ethiopia

${ }^{3}$ Telethon Kids Institute, Nedlands, Western Australia, Australia

${ }^{4}$ Agriculture and Environment, The University of Western Australia, Crawley, Western Australia, Australia

Correspondence to Alemken Jegnie; alemken.jegnie@research.uwa. edu.au

\section{ABSTRACT}

Introduction Multidrug-resistant tuberculosis (MDR-TB) is a common public health problem affecting pregnant women. However, the impact of MDR-TB and its medication on pregnancy and perinatal outcomes has been poorly understood and inconsistently reported. Therefore, using the available literature, we aim to determine whether MDR-TB and its medications during pregnancy impact maternal and perinatal outcomes.

Methods and analysis This systematic review and meta-analysis will adhere to the Preferred Reporting Items for Systematic Reviews and Meta-Analyses guidelines. Systematic searches will be conducted in PubMed, Scopus and Web of Science on 10 February 2020 for studies that reported adverse maternal and perinatal outcomes due to MDR-TB and/or its medication. The search will be performed without language and time restrictions. Adverse birth outcomes include miscarriage or abortion, stillbirth, preterm birth, low birth weight, small and large for gestational age, and neonatal death. Two independent reviewers will screen search records, extract data and assess the quality of the studies. The Newcastle-0ttawa Quality Assessment Scale will be used to assess the methodological quality of the included studies. In addition to a narrative synthesis, a random-effects meta-analysis will be conducted when sufficient data are available. $I^{2}$ statistics will be used to assess the heterogeneity between studies.

Ethics and dissemination As it will be a systematic review and meta-analysis based on previously published evidence, there will be no requirement for ethical approval. Findings will be published in a peer-reviewed journal and will be presented at various conferences.

\section{INTRODUCTION}

Tuberculosis (TB) is a common non-obstetric cause of death among pregnant women and one of the risk factors for adverse birth outcomes. ${ }^{1}$ It has been estimated that more than 200000 pregnant women are affected by TB globally, with $41 \%$ and $31 \%$ of the cases occurring in African and South-East Asian countries, respectively. ${ }^{2}$ The emergence of
Strengths and limitations of this study

- To our knowledge, this systematic review will be the first to synthesise and quantify the impact of multidrug-resistant tuberculosis and its medication on adverse birth and maternal outcomes.

- Databases will be searched without time restrictions and independent evaluation will be employed.

- A recently developed robust variance meta-analysis technique will be applied to detect and correct for publication bias.

- The potential limitation of this review could be the heterogeneity of studies in outcomes of interest.

multidrug-resistant tuberculosis (MDR-TB) with resistance to rifampicin and isoniazid (the two most important first-line therapeutic agents) and extensively resistant tuberculosis (XDR-TB), with additional resistance to a fluoroquinolone and a second-line injectable drug, has become a major global concern that poses additional challenges for the treatment of TB among pregnant women. ${ }^{3}$ In 2017, globally, there were an estimated 490000 incident MDR-TB cases, of which $9 \%$ were XDR-TB cases. $^{4}$

MDR-TB is common among pregnant women and may result in a higher risk of pregnancy-related complications and perinatal death. ${ }^{15}$ It has also been suggested that MDR-TB during pregnancy could potentially trigger an increased risk of adverse birth outcomes such as spontaneous abortion, small for gestational age and low birth weight. ${ }^{6-8}$ The impact of MDR-TB in pregnant women can be aggravated by several factors, such as the severity of the disease, the site of infection and the treatment regimen, and substantially varies from mild symptoms to severe complications and sometimes death. ${ }^{59}$ Pregnant women with untreated MDR-TB are 
at increased risks of maternal and infant mortality, suggesting treatment with second-line TB drugs. ${ }^{10}$ However, as the treatment of MDR-TB takes longer duration and is more toxic than Drug-susceptible Tuberculosis (DS-TB), the risk of adverse birth outcomes such as miscarriage, stillbirth, preterm birth and low birth weight is suggested to be higher in patients with MDR-TB than in patients with DS-TB. ${ }^{11}$ These adverse birth outcomes often occur as a result of the disease process itself or due to side effects related to second-line TB medications such as fluoroquinolones, aminoglycosides, ethionamide and prothionamide. Some of these drugs have been identified to have teratogenic effects. For example, aminoglycosides, including streptomycin, kanamycin and amikacin, have been shown to impact fetal birth weight and hearing capacity. ${ }^{61213}$ As a result, some studies have recommended termination of pregnancy, ${ }^{814}$ and others have suggested reducing the dose of teratogenic drugs or suspending the treatment during pregnancy. ${ }^{15} 16$ On the contrary, some studies did not find an association between MDR-TB medications and perinatal outcomes. ${ }^{17}$ However, previous studies have provided such conflicting evidence based on individual studies with a small sample size, ${ }^{818} 19$ and adequate data regarding the impact of second-line MDR-TB drugs in pregnant women are lacking. ${ }^{14}$ In addition, to the best of our knowledge, no MDR trial currently conducted worldwide includes pregnant patients, which presents a major obstacle to developing guidance of what MDR-TB drugs are safe and effective in pregnancy.

As most previous studies revealed inconsistent results, mainly because of limited statistical power, a comprehensive systematic review including meta-analysis is required to have clarity regarding the impact of MDR-TB and secondline TB drugs on perinatal outcomes. Quantification of the effects of MDR-TB and its medication on birth outcomes is essential to inform service providers and policymakers in allocating resources and in the prevention of adverse birth outcomes in countries where MDR-TB is prevalent.

\section{Objective}

The aim of this systematic review and meta-analysis is to assess the impact of MDR-TB and its medications during pregnancy on adverse maternal and birth outcomes.

\section{METHODS}

\section{Search strategy}

This systematic review and meta-analysis will adhere to the Preferred Reporting Items for Systematic Reviews and Meta-analyses guidelines. ${ }^{20}$ Systematic searching will be conducted in PubMed, Scopus and Web of Science databases to identify all potential studies that reported adverse maternal and perinatal outcomes among pregnant women who had MDR-TB diagnosis or exposure to MDR-TB medications during pregnancy. The search will be conducted from the inception of each database to 10 February 2020, without language and time restrictions. The overall study will be conducted from 14 December
2019 to 21 March 2020. Medical subject heading terms and combinations of keywords related to pregnancy, MDR-TB and MDR-TB medications, and pregnancy outcomes will be used for the search. A complete search strategy for the PubMed database is available in online supplementary table S1. The reference lists and citations of the retrieved articles will be checked manually for additional studies. The authors of the papers will be contacted through email when there is a need for additional information.

\section{Eligibility criteria}

\section{Inclusion criteria}

Studies will be included in the systematic review if they evaluate any maternal morbidity and mortality as well as perinatal adverse outcomes among pregnant women with MDR-TB (with or without MDR-TB medications). The selection criteria to identify potential studies will be study population (pregnant women), intervention (MDR-TB and/or its medication during pregnancy), comparator (pregnancies with no MDR-TB, pregnancies with MDR-TB not receiving treatment) and outcomes (adverse perinatal outcomes, and maternal morbidity and mortality). MDR-TB medications will be defined, according to the recent WHO guideline on drug-resistant TB treatment, as second-line TB agents that are recommended for the treatment of drug-resistant TB. ${ }^{21}$ These agents include levofloxacin, moxifloxacin, bedaquiline, linezolid, clofazimine, cycloserine, terizidone, delamanid, imipenem-cilastatin, meropenem, amikacin, ethionamide, prothionamide and p-aminosalicylic acid.

\section{Exclusion criteria}

We will exclude correspondence, reviews, editorials and conference abstracts. Studies conducted only on drugsusceptible TB or on animals will also be excluded. When multiple studies used the same data, we will include the study with the most detailed clinical data, with the largest sample size or with the longest follow-up period to avoid duplication.

\section{Outcomes}

The review includes both perinatal adverse outcomes, and maternal morbidity and mortality. We will include studies that reported outcomes of pregnancies complicated by MDR-TB and non-drug-resistant TB to construct risk ratios for each study and look for a pooled risk ratio. Table 1 shows the definition of the perinatal adverse outcomes and the maternal morbidity and mortality outcomes of the study. The outcomes of the study will be recorded as prevalence, incidence, relative risks or ORs, as reported in the individual papers.

\section{Data extraction}

All identified articles from the systematic searching will be uploaded into Rayyan (https://rayyan.qcri.org). Two researchers (KAA and $\mathrm{AJ}$ ) will independently screen the titles and abstracts of the studies and will then review the full text based on the eligibility criteria. The two researchers will compare the results and disagreements will be resolved 
Table 1 Definitions of adverse maternal and perinatal outcomes of the systematic review

\begin{tabular}{ll}
\hline Outcomes & Definitions of outcomes \\
\hline Preterm & Birth before 37 completed weeks' gestation. \\
Low birth weight & Birth weight less than $2500 \mathrm{~g}$. \\
Small for gestational age & Birth weight $<10$ th percentile for gestational age. \\
Severe growth restriction & Birth weight <3rd percentile for gestational age. \\
Stillbirth & An infant born with no signs of life at 20 or more weeks' gestation. \\
Abortion & Termination of a pregnancy before 20 weeks' gestation. \\
Congenital anomalies & Any major birth defect or as defined by individual studies. \\
Neonatal mortality & Death of a liveborn infant in the first 28 days of life. \\
Maternal morbidity & Any pregnancy and birth complications reported in the original studies. \\
Maternal mortality & Death while pregnant or within 42 days of the end of pregnancy.
\end{tabular}

through discussion. If consensus is not reached between these two researchers, disagreements will be resolved by discussion with a third investigator (AAA).

Data from the included studies will be extracted and compiled using a standardised Excel spreadsheet. We will extract information from each study on the last name of the first author, year of data collection and publication, report type (grey literature vs published studies), study country, study design and data source. Information will also be collected on maternal age, sample size, effect size as reported by a study, multiple pregnancies, type of pregnancy outcomes and the number of cases with adverse birth outcomes. When available, the following additional information will also be extracted from the primary studies: percentage of resistance to particular TB medicines, duration of MDR-TB treatment in months, percentage of pregnant women with HIV infection and percentage of pregnant women with diabetes mellitus. Moreover, we will make an effort to include relevant information unavailable to the original study, such as socioeconomic setting (eg, poor or rich country, the income level for each country, World Bank (WB) member or not) and geographical dimension (the state/province where the study is conducted). A data extraction sheet is available in online supplementary table S2.

\section{Quality and bias assessment}

The methodological quality of the included studies will be assessed independently by the same two investigators (KAA and AJ) using a modified version of the NewcastleOttawa Quality Assessment Scale. ${ }^{22}$ This tool has scores ranging from 0 to 9 ; scores between 1 and 4 will be defined as low quality, scores between 5 and 7 will be defined as medium quality, and scores between 8 and 9 will be defined as high quality. Publication bias will be assessed graphically by a funnel plot and statistically using a recently developed robust variance estimation (RVE) technique. ${ }^{2324}$

\section{Data analysis}

A systematic narrative synthesis will be conducted to describe the outcomes of the study. When two or more studies are available, a random-effects meta-analysis will be used to obtain a pooled estimate value for each of the outcomes of interest. Heterogeneity between studies will be examined using the Cochran's $Q$ test and quantitatively measured by the index of heterogeneity squared $\left(\mathrm{I}^{2}\right)$ statistics and its $95 \% \mathrm{CI}^{25}$ Heterogeneity will be considered low, moderate and high when $\mathrm{I}^{2}$ values are below $25 \%$, between $25 \%$ and $75 \%$, and above $75 \%$, respectively. ${ }^{25}$ When there is evidence of significant heterogeneity, the sources of this will be explored through meta-regression using study characteristics as covariates. The Hedges $e t a l^{26}$ and Tipton ${ }^{27}$ small-sample corrected RVE method will be applied to perform the meta-regression; this approach handles non-independent effect sizes without knowledge of the within-study covariance structure. Unlike the traditional meta-regression approaches, the RVE method has some unique benefits, such as the following: (1) the coefficients are consistent estimates of the underlying population parameters under a broad set of conditions including non-normality; (2) the results do not need the predictor variables to be fixed; and (3) RVE yields valid SEs, point estimates, CIs and significance tests when effect sizes are non-independent, without requiring to model the exact nature of this dependence. ${ }^{2728}$ Hedges $e t a l^{26}$ show that the RVE approach performs well when the number of studies is large. However, Tipton ${ }^{27}$ made small-sample adjustments to both the RVE estimator and df, and it has been suggested that the RVE method can also perform well when the number of studies is small, as few as 10. An inverse variance weighting will be used to provide asymptotically accurate estimates of SEs and valid inferences. This approach is distribution-free, provides valid point estimates and SEs, and performs an appropriate hypothesis test even when the degree and structure of dependence between effect sizes are unknown; hence, the statistical inferences made will be unbiased and correct.

\section{Patient and public involvement}

No patient will be involved in the study. 


\section{DISCUSSION AND CONCLUSION}

This comprehensive systematic review will quantify the impacts of MDR-TB and second-line TB medication on adverse maternal and birth outcomes such as prematurity, low birth weight and small for gestational age, and various other obstetrical and perinatal outcomes. The results will provide comprehensive information essential for healthcare providers and policymakers to better understand the impact of MDR-TB and its medication on adverse maternal and birth outcomes, and to design appropriate treatment regimen and follow-up for pregnant women with MDR-TB. This review also identifies research gaps in the literature regarding the subject and provides a basis for future studies.

\section{ETHICS AND DISSEMINATION}

There will be no requirement for ethical approval, and the findings will be published in the peer-reviewed journal and will be presented at various conferences.

\section{AMENDMENTS OF THE PROTOCOL}

If there is a need to amend this protocol, the date of each amendment and the reason for the change will be described.

Contributors KAA, AAA and AJ conceived of the study, developed the search strategy and drafted the protocol. All authors critically revised the manuscript for methodological and intellectual content and have read and approved the final manuscript.

Funding The authors have not declared a specific grant for this research from any funding agency in the public, commercial or not-for-profit sectors.

Competing interests None declared.

Patient consent for publication Not required.

Ethics approval As this will be a systematic review and meta-analysis based on previously published evidence, there will be no requirement for ethical approval.

Provenance and peer review Not commissioned; externally peer reviewed.

Open access This is an open access article distributed in accordance with the Creative Commons Attribution Non Commercial (CC BY-NC 4.0) license, which permits others to distribute, remix, adapt, build upon this work non-commercially, and license their derivative works on different terms, provided the original work is properly cited, appropriate credit is given, any changes made indicated, and the use is non-commercial. See: http://creativecommons.org/licenses/by-nc/4.0/.

ORCID iD

Alemken Jegnie http://orcid.org/0000-0002-2083-4585

\section{REFERENCES}

1 Zumla A, Bates M, Mwaba P. The neglected global burden of tuberculosis in pregnancy. Lancet Glob Health 2014;2:e675-6.
2 Sugarman J, Colvin C, Moran AC, et al. Tuberculosis in pregnancy: an estimate of the global burden of disease. Lancet Glob Health 2014;2:e710-6.

3 Gandhi NR, Nunn P, Dheda K, et al. Multidrug-Resistant and extensively drug-resistant tuberculosis: a threat to global control of tuberculosis. The Lancet 2010;375:1830-43.

4 WHO. Global tuberculosis report 2017. Geneva: World Health Organization, 2017.

5 Khan M, Pillay T, Moodley J, et al. Pregnancies complicated by multidrug-resistant tuberculosis and HIV co-infection in Durban, South Africa. Int J Tuberc Lung Dis 2007;11:706-8.

6 Asuquo B, Vellore AD, Walters G, et al. A case-control study of the risk of adverse perinatal outcomes due to tuberculosis during pregnancy. J Obstet Gynaecol 2012;32:635-8.

7 Espiritu N, Jave O, Kirwan DE, et al. Congenital transmission of multidrug-resistant tuberculosis. Am J Trop Med Hyg 2014;91:92-5.

8 Palacios E, Dallman R, Muñoz M, et al. Drug-resistant tuberculosis and pregnancy: treatment outcomes of 38 cases in Lima, Peru. Clin Infect Dis 2009;48:1413-9.

9 Tabarsi P, Baghaei P, Mirsaeidi M, et al. Multi-drug resistant tuberculosis in pregnancy: need for more intensive treatment. Infection 2007;35:477-8.

10 Lange C, Dheda K, Chesov D, et al. Management of drug-resistant tuberculosis. The Lancet 2019;394:953-66.

11 Dudnyk A, Pavel'chuk O. Multidrug-resistant tuberculosis in pregnant women: treatment and birth outcomes. Eur Respiratory Soc 2016;48.

12 Mathad JS, Gupta A. Tuberculosis in pregnant and postpartum women: epidemiology, management, and research gaps. Clin Infect Dis 2012;55:1532-49.

13 Hong H, Dooley KE, Starbird LE, et al. Adverse outcome pathway for aminoglycoside ototoxicity in drug-resistant tuberculosis treatment. Arch Toxicol 2019;93:1385-99.

14 Lange C, Abubakar I, Alffenaar J-WC, et al. Management of patients with multidrug-resistant/extensively drug-resistant tuberculosis in Europe: a TBNET consensus statement. Eur Respir J 2014;44:23-63.

15 Nitta AT, Milligan D. Management of four pregnant women with multidrug-resistant tuberculosis. Clin Infect Dis 1999;28:1298-304.

16 Loto OM, Awowole I. Tuberculosis in pregnancy: a review. $J$ Pregnancy 2012;2012:1-7.

17 Dheda K, Gumbo T, Maartens G, et al. The epidemiology, pathogenesis, transmission, diagnosis, and management of multidrug-resistant, extensively drug-resistant, and incurable tuberculosis. Lancet Respir Med 2017;5:291-360.

18 Shin S, Guerra D, Rich M, et al. Treatment of multidrug-resistant tuberculosis during pregnancy: a report of 7 cases. Clin Infect Dis 2003;36:996-1003.

19 Drobac PC, del Castillo H, Sweetland A, et al. Treatment of multidrug-resistant tuberculosis during pregnancy: long-term followup of 6 children with intrauterine exposure to second-line agents. Clin Infect Dis 2005;40:1689-92.

20 Moher D, Liberati A, Tetzlaff J. Preferred reporting items for systematic reviews and meta-analyses: the PRISMA statement. Ann Intern Med 2009;151:264-9.

21 WHO. WHO consolidated guidelines on drug-resistant tuberculosis treatment. Geneva, 2019.

22 Wells G, Shea B, O'Connell D, et al. Newcastle-Ottawa quality assessment scale cohort studies, 2014.

23 Ioannidis JPA, Stanley TD, Doucouliagos $\mathrm{H}$. The power of bias in economics research. Oxford, UK: Oxford University Press, 2017: F236-65.

24 Stanley TD, Doucouliagos H. Meta-Regression approximations to reduce publication selection bias. Res Synth Methods 2014;5:60-78.

25 Higgins JPT, Thompson SG. Quantifying heterogeneity in a metaanalysis. Stat Med 2002;21:1539-58.

26 Hedges LV, Tipton E, Johnson MC. Robust variance estimation in meta-regression with dependent effect size estimates. Res Synth Methods 2010;1:39-65.

27 Tipton E. Small sample adjustments for robust variance estimation with meta-regression. Psychol Methods 2015;20:375-93.

28 Fisher Z, Tipton E. robumeta: an R-package for robust variance estimation in meta-analysis, 2015. 